\title{
Development and characterization of a $\mathrm{ZnO} / \mathrm{Ge}$ photodiode for optical radiation measurements in the near infrared
}

\author{
Zahra Ben Achour ${ }^{1, *}$, Marwa Hammami ${ }^{2}$, and Oualid Touayar ${ }^{1}$ \\ ${ }^{1}$ Metrology Team of Radiometric and Pyrometric Measurements, Carthage University, National Institute of Applied \\ Science and Technology, INSAT BP676 Centre Urbain Nord, 1080 Tunis Cedex, Tunisia \\ ${ }^{2}$ University Campus Farhat Hachad, Tunis, El Manar University, Faculty of Sciences of Tunis, Tunis, Tunisia
}

Received: 19 December 2016 / Accepted: 2 May 2017

\begin{abstract}
The metrology group of radiometric and pyrometric measurements of INSAT include among others the development of detectors that will be used as a standard in the MMA Laboratory for optical radiation measurements in the visible and infrared spectral range. In this work, we present the design and realization of a detector for near infrared radiation measurements: it is a photodiode $\mathrm{ZnO} / \mathrm{Ge}$ based on a germanium junction and a thin layer of zinc oxide. Then we electrically and optically characterized the photodiode thus realized, for which we developed an energy band diagram. The obtained results have allowed us to note an improvement in the optical and electrical characteristics of the $\mathrm{ZnO} / \mathrm{Ge}$ photodiode, compared to those performed in our laboratory and based on single Germanium. The reflectivity is reduced by about $9 \%$ for the wavelength range of $800 \mathrm{~nm}$ to $2000 \mathrm{~nm}$. The shunt resistance increases from $95 \Omega$ to $12.915 \mathrm{k} \Omega$. However, the series resistance increases from $1.08 \Omega$ to $36 \Omega$ but it is still an acceptable value. The proposed energy band diagram explains the charge carrier transport phenomena for our structure and it is in good agreement with experimental results.
\end{abstract}

Keywords: photodiode / zinc oxide / transfer detector / reflectivity / radiometric measurements

\section{Introduction}

Research work carried out within the metrology group of radiometric and pyrometric measurements of INSAT, have achieved NP junctions by ion implantation from Ge p-type substrate and the realization of porous germanium photodiodes [1-3]. These studies have shown an improvement in the absorption of the incident light with respect to the commercial photodiodes based on the junction germanium.

The only devices to minimize the reflection coefficient are trap detectors requiring the use of several photodiodes placed in well-defined geometric configurations $(3-5$ photodiodes). Our objective is to realize a detector with a low reflection coefficient using a single photodiode, hence the interest of optical characterization.

In the current work, we study the influence of a thin $\mathrm{ZnO}$ layer deposited onto a Ge junction. Indeed, the $\mathrm{ZnO}$ thin films in addition to their transparent features in the near infrared, are excellent candidates for the transparent electrodes and antireflective coats [3-5]. The optical and electrical properties of $\mathrm{ZnO}$ thin films allow them to reduce

\footnotetext{
* Corresponding author: zbenachour@yahoo.fr
}

the reflectivity of the substrates on which they are deposited and to drive the charges created on the surface to the outer circuit [6].

The spectral sensibility of the photodiodes is proportional to the reflexion coefficient and the internal quantum efficiency (Eq. (1)) which itself depends on the diffusion constant of the charge carriers in the semiconductors $[1,6]$.

$$
S=e \eta(1-\rho) \frac{\lambda}{h c},
$$

with $\rho$ : reflexion coefficient; $\eta$ : internal quantum efficiency; $\lambda$ : wave length; $h$ : Planck constant; $c$ : light speed; $e$ : electron charge.

The improvement of the spectral sensitivity is achieved by reducing the reflection coefficient and by increasing the internal quantum efficiency varying in the same way as the diffusion constant of the semiconductor.

In order to have a large diffusion constant, it is necessary to have a low recombination rate of the charge carriers. This is ensured only by low series resistance and high shunt resistance.

In order to evaluate the quality of our photodiodes, we will proceed to their electrical characterization. This will allows us to evaluate the different electrical characteristics 


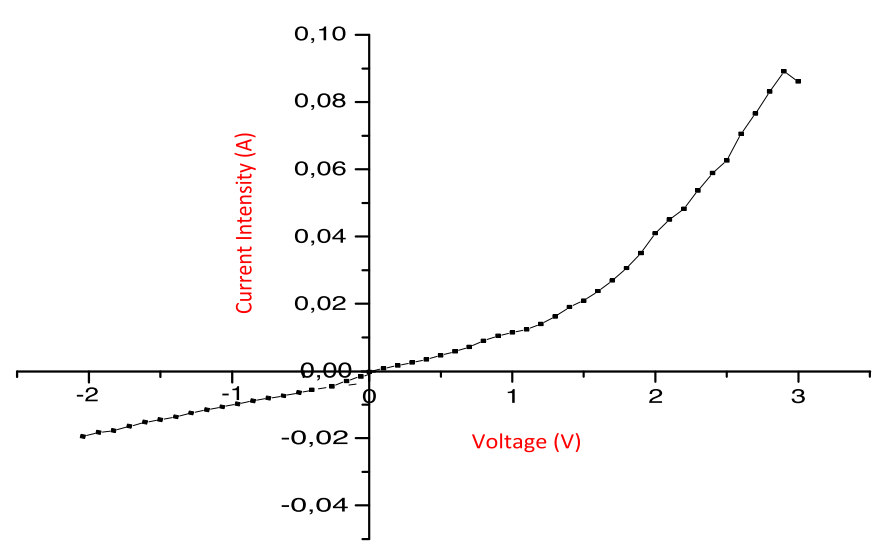

Fig. 1. $I-V$ characteristic of the germanium junctions.

of our devices and the direction of their evolution in order to compare them with commercially available standard photodiodes.

In the first part of this paper we present the results of electrical and optical characterization of germanium junctions.

In the second part we are interested in the characterization of the new photodiode type $\mathrm{ZnO} / \mathrm{Ge}$ that we developed. These photodiodes are Germanium junctions formed by ion implantation where we deposited on the front face a thin layer of zinc oxide by the Spray pyrolysis technique $[4,7,8]$.

\section{NP germanium junctions characterization}

The used NP germanium junctions are made by ion implantation from germanium polymer substrate type $\mathrm{P}$. This P-type substrate is initially doped gallium with $(1,0,0)$ orientation, a resistivity lower than $1 \Omega \mathrm{cm}$ and a thickness of $300 \mu \mathrm{m}$. These substrates have a reflectivity of about $33 \%$ for the wavelength range between $800 \mathrm{~nm}$ and $2000 \mathrm{~nm}$ $[1,2]$. Ion implantation is performed by the ion implanter installed in Microelectronics Institute of Barcelona in Spain. It is performed with an ionizing beam energy of $150 \mathrm{keV}$ for phosphorus impurity doses of $1 \times 10^{-15} \mathrm{~atm} /$ $\mathrm{cm}^{2}[1,9,10]$. The realized junctions have a junction depth of about $0.4 \mu \mathrm{m}$. The metallic contacts are made by thermal evaporation, with gold covering the entire backside and gold circle electrode on the front side of the junctions.

\subsection{Electrical characterization of germanium junctions}

The $I-V$ characteristic shows the internal processes that regulate the operation of the photodiode. It also represents the macroscopic characteristics $R_{s}, R_{\mathrm{sh}}, I_{\mathrm{ph}}$ and the current diodic showing the transport phenomena of the charge carriers within the structure. The series resistance $R_{s}$ of a photodiode arises from the resistance of the contacts and the resistance of the undepleted germanium. It therefore models the carriers transport in the depletion zone. As for the shunt resistance $R_{\mathrm{sh}}$, it models the current leakage and charge carriers in the depletion zone. The $I_{\mathrm{ph}}$ is the photonic current of the photodiode.
Table 1. Electrical parameters of germanium junctions.

\begin{tabular}{ll}
\hline Threshold voltage, $V_{s}$ & $0.411 \mathrm{~V}$ \\
Photonic current, $I_{\mathrm{ph}}$ & $-8.52 \times 10^{-5} \mathrm{~A}$ \\
Saturation current, $I_{\text {sat }}$ & $1.06 \times 10^{-4} \mathrm{~A}$ \\
Series resistance, $R_{s}$ & 1.08 \\
Shunt resistance, $R_{\mathrm{sh}}$ & 94.98 \\
\hline
\end{tabular}

The $I-V$ characteristic in darkness is detected on a measurement bench where the photodiode are biased by a voltage supplied by a DC power supply.

The method of determining the electrical parameters of the photodiode has been widely detailed in several publications $[1,11]$. This method is based on the tracing of the corresponding characteristics $\operatorname{Ln}(I)=f(V)$ from the experimental responses $I-V$. The advantage of studying the characteristics $\operatorname{Ln}(I)=f(V)$ is to show efficiently the different modes of conduction, to be able to localize them according to the voltage and to determine approximately the saturation current and the series and shunt resistances.

According to the $I-V$ characteristic curve in Figure 1, we deduce the photonic current corresponding to our germanium junctions. Exploiting this characteristic also allowed us to determine the values of the series resistance and the shunt resistance. Table 1 was synthesized various electrical parameters of the germanium junctions.

The series resistance is similar to those of commercial photodiodes of few ohms. The series resistance low value of our device, highlights the quality of the internal structure. Indeed, the ion implantation is a doping process that results in a uniform distribution of implanted impurities. Furthermore, the post-implantation treatment can cure defects appeared during implantation which reduced the traps of the charge carriers and thus the series resistance.

The shunt resistance of the junctions is lower than that of available commercial photodiodes which have a shunt resistance of a few hundred kilo ohms. Indeed, this resistance characterizes losses by recombination of the carriers due to the germanium structural defects. The existence of cracks and structural complex defects in surface becomes the seat of physical phenomenon similar to a parallel resistor $R_{\mathrm{sh}}[9,10]$.

\subsection{Optical characterization of the germanium junctions}

The reflectivity determination allows us to evaluate the level of reflection loss of the incident luminous flux on the Ge/Air interface of the device forming the photodiode. The measurements were performed at the Laboratory of Physics Condensed Matter (LPMC) of the Faculty of Sciences of Tunis with a spectrophotometer Perkin Elmer Lambda 950. The spectral range extended from $200 \mathrm{~nm}$ to $2500 \mathrm{~nm}$.

Figure 2 represents the results of the reflectivity measured on the photodiodes based on germanium junction.

The reflectivity of germanium junctions is about $30 \%$ within the wavelength range from $800 \mathrm{~nm}$ to $2000 \mathrm{~nm}$. We note also a slight reduction in the reflectivity of around $3 \%$ between germanium junctions and the base bare germani- 


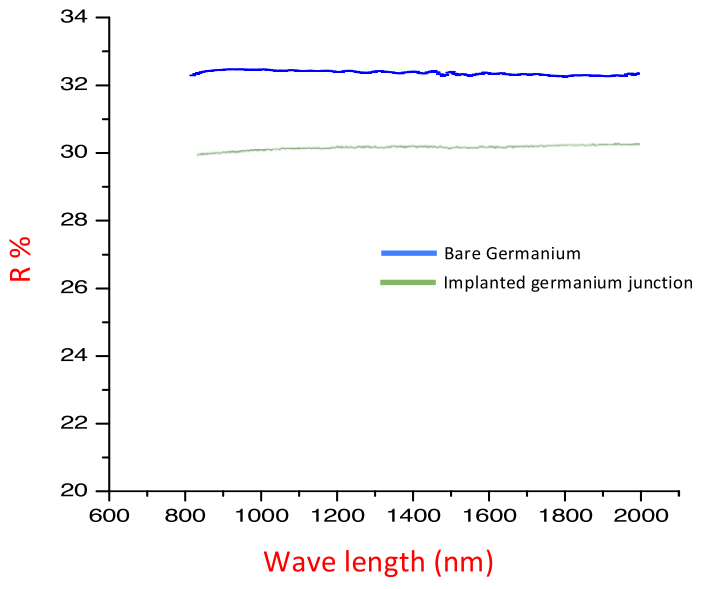

Fig. 2. Reflectivity of germanium junctions.

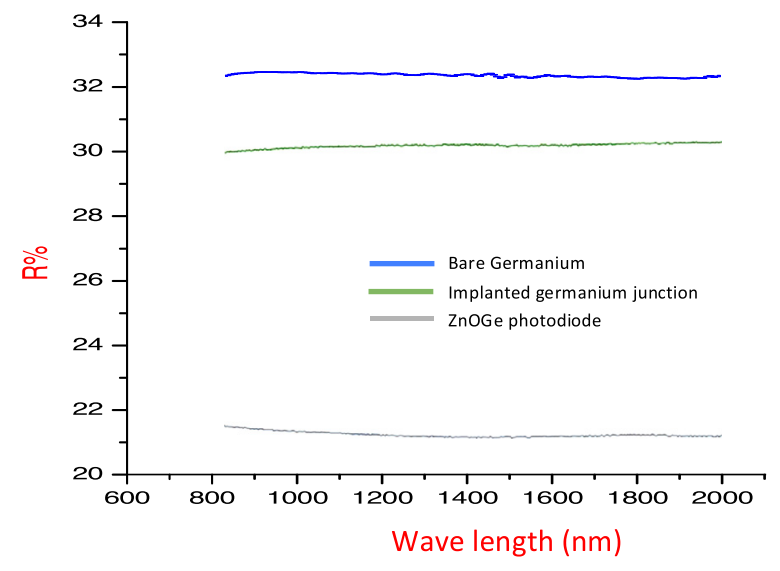

Fig. 4. Reflectivity of the $\mathrm{ZnO} / \mathrm{Ge}$ photodiode.

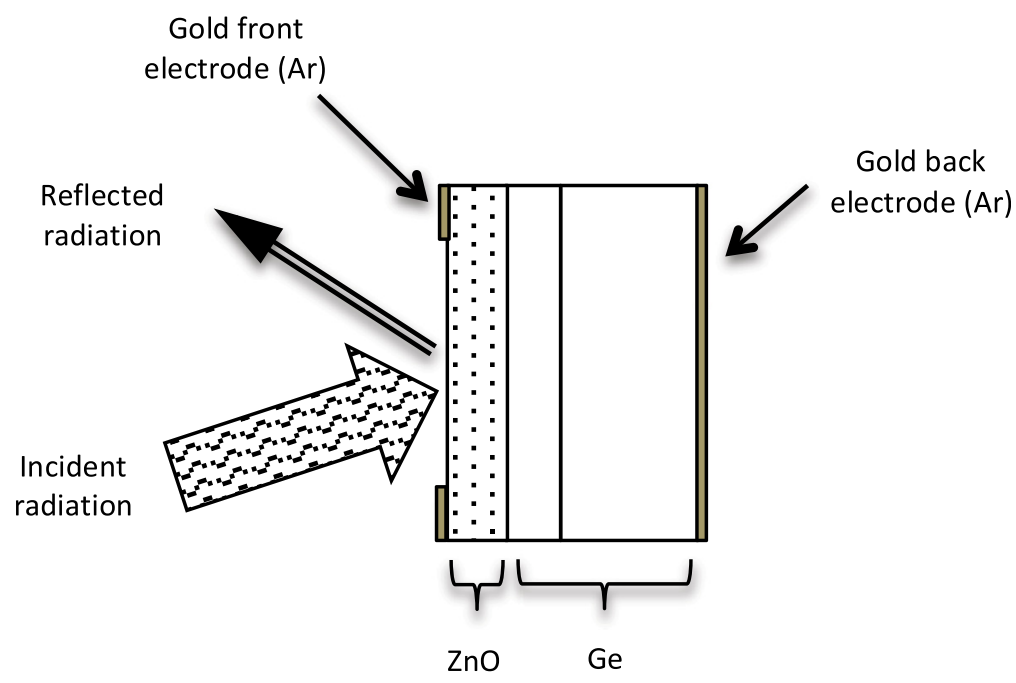

Fig. 3. Structure of the $\mathrm{ZnO} / \mathrm{Ge}$ photodiode.

um. This reflectivity reduction is partly due to the native oxide on the surface of the germanium substrate, in addition to the oxidation of germanium during the postimplantation treatment. Indeed, ion implantation generates a crystallographic disorder at the implanted matrix, thereby requiring an annealing which will cure the defects induced by implantation to activate the implanted impurities and homogenize the doped layer. The analysis of results allows us to deduce that after optimization of manufacturing conditions of the junctions, it is imperative to introduce antireflection layers in the structure of germanium photodiodes to reduce reflectivity. These layers enable to reduce the reflectivity and in the other hand chemically stabilize the surface of the germanium and preserve its physical and chemical characteristics.

\section{$3 \mathrm{ZnO}$ deposition on $\mathrm{Ge}$ junctions: optical and electrical characterization}

The $\mathrm{ZnO}$ thin films are deposited by spray pyrolysis technical in inert atmosphere from a $0.1 \mathrm{M}$ zinc acetate solution $3 \%$ doped with aluminum. The deposition temperature is $460{ }^{\circ} \mathrm{C}$ for optimal duration of $60 \mathrm{~min}$ [4]. The choice of this temperature allows to obtain $90 \%$ transparent $\mathrm{ZnO}$ layers in the range of 500-2500 nm. The $\mathrm{ZnO}$ thin films thus produced have a resistivity of $3 \times 10^{-3} \Omega \mathrm{cm}$ and a preferred crystallographic orientation $(0,0,2)$ [4]. The thickness of these thin films is approximately $1 \mu \mathrm{m}$ and their gaps is of the order of $3.21 \mathrm{eV}$ [4]. Figure 3 shows a section of the structure forming the achieved $\mathrm{ZnO} / \mathrm{Ge}$ photodiode.

\subsection{Optical characterization of the $\mathrm{ZnO} / \mathrm{Ge}$ structure}

We are interested in this part to the study of the reflectivity in the spectral range from 800 to $2000 \mathrm{~nm}$, of the new structure the $\mathrm{ZnO} / \mathrm{Ge}$ photodiode. The results of this study made on the same spectrometer previously used (Sect. 2.2), are represented in Figure 4.

We find that the $\mathrm{ZnO}$ layers bring a reduction in the reflectivity of Ge junctions. In fact, the reflectivity passes from an average value of $30-21 \%$ over a wavelength range between 800 and $2000 \mathrm{~nm}$ which is therefore a gain of about $9 \%$ for the light absorption. This gain is due to the antireflective feature of the $\mathrm{ZnO}$ thin films deposited on the 


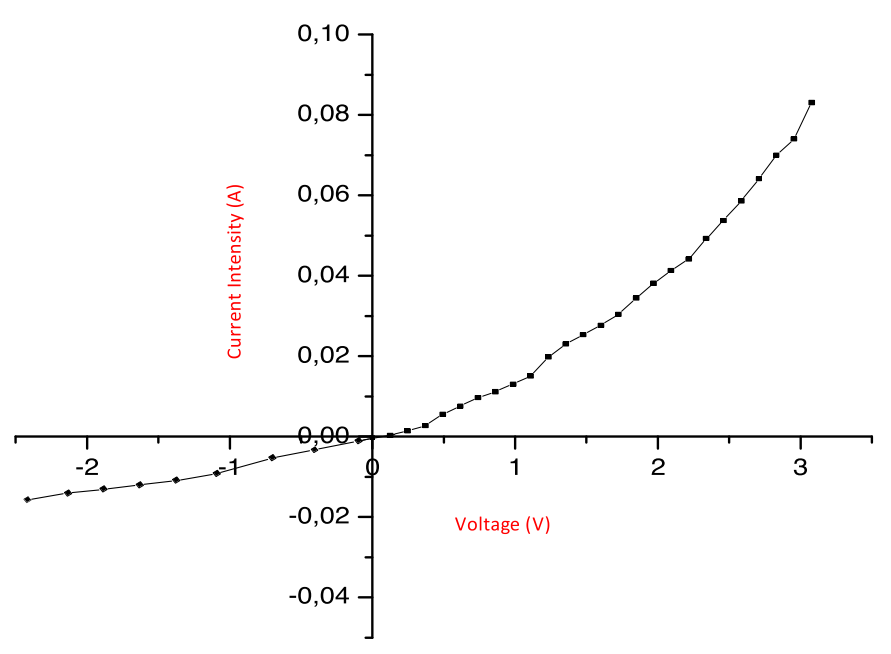

Fig. 5. $I-V$ characteristic of the $\mathrm{ZnO} /$ Ge photodiode.

germanium junction. Indeed, it has been demonstrated that the thin layers of $\mathrm{ZnO}$ thus deposited have a reflectivity level on average equal to $20 \%$ in the spectral range from 400 to $1800 \mathrm{~nm}$ [4].

\subsection{Electrical characterization of the $\mathrm{ZnO} / \mathrm{Ge}$ structure}

For this part we have adopted the same approach described above. Figure 5 we represent the $I-V$ characteristic of the $\mathrm{ZnO} / \mathrm{Ge}$ photodiode.

According to this graph, it can be deduced that the structure of the germanium photodiode covered with a thin $\mathrm{ZnO}$ layer on its front face, has a series resistance $R_{s}$ and a shunt resistance $R_{\mathrm{sh}}$, higher than the photodiode formed by a germanium bare junction. Indeed, the slope $\Delta I / \Delta I(V$ $\rightarrow 0$ ) decreases significantly, resulting in a decrease in leakage current and a significant increase in the shunt resistance.

The characteristic parameters of the $\mathrm{ZnO} / \mathrm{Ge}$ photodiode are summarized in Table 2.

When the germanium photodiode is provided with a thin layer of $\mathrm{ZnO}$, there is a clear improvement in its current response following the change in voltage which reflects the new values of shunt and series resistances we could get.

The improvement of the $I-V$ characteristic after introduction of the $\mathrm{ZnO}$ layer in the photodiode structure is due to the optical and electrical characteristics of this layer. Indeed, the antireflection appearance of the $\mathrm{ZnO}$ layer contributed to the optic flux confinement which improves the absorption of the photodiode and hence its response. The photonic current determined from the $I-V$ characteristic under illumination is in the order of $-1.22 \times 10^{-5} \mathrm{~A}$.

The considerable increase in the shunt resistance from $94.98 \Omega$ to $12.915 \mathrm{k} \Omega$ after the thin $\mathrm{ZnO}$ layer deposition is due to the passivation of dangling bonds of the germanium as well as the native oxide. This therefore leads to a reduction of the quantum wells as well as of imperfections where the charge carriers are trapped especially in surface. Besides, the $\mathrm{ZnO}$ thin films are transparent electrodes that
Table 2. Electrical parameters of the $\mathrm{ZnO} / \mathrm{Ge}$ photodiode.

\begin{tabular}{ll}
\hline Threshold voltage, $V_{s}$ & $0.471 \mathrm{~V}$ \\
Photonic current, $I_{\mathrm{ph}}$ & $-1.22 \times 10^{-5} \mathrm{~A}$ \\
Saturation current, $I_{\mathrm{sat}}$ & $1.8 \times 10^{-4} \mathrm{~A}$ \\
Series resistance, $R_{s}$ & 36 \\
Shunt resistance, $R_{\mathrm{sh}}$ & $12.915 \mathrm{k}$ \\
\hline
\end{tabular}

recover the charges created on the surface. This has the effect of reducing the recombination velocity of charge carriers and to extend their lifetime in surface.

Furthermore, there is an increase of the series resistance which characterizes the internal resistance. The series resistance changes from a value of $1.08-36 \Omega$. This variation is probably due to the thermal agitation effects on the internal structure of the junction after annealing during deposition of the $\mathrm{ZnO}$ layer. We can also attribute this increase in the potential barrier that is created at the interface between $\mathrm{ZnO}$ layer and the germanium surface. Indeed, the gap of germanium is $0.66 \mathrm{eV}$ [1] and that of the deposited $\mathrm{ZnO}$ layer is of the order of $3.21 \mathrm{eV}$ [4].

\section{Energy bands diagram}

To understand the conversion process of the charge carriers and explain the improvement made by depositing $\mathrm{ZnO}$ thin films on germanium photodiodes, we propose a diagram of the energy bands of the $\mathrm{ZnO} / \mathrm{Ge}$ structure thus formed. The physical model of the energy bands diagram that we propose is a model explaining the mechanisms of electrical conduction within our photodiode and it takes into account the different layers and their physical properties.

Our studied structure is a heterojunction in which two different types of semiconductor are brought into contact. Therefore they exchange electrons so as to align their Fermi levels. This exchange takes place near the junction thereby creating a space charge zone which is associated with a potential barrier $V_{d}$ which stops the carrier diffusion and defines the state of equilibrium.

$$
V_{d}=V_{2}-V_{1}=\phi_{1}-\phi_{2},
$$

where $V_{1}$ and $V_{2}$ : potentials of the two semiconductors and $e \phi_{1}, e \phi_{2}$ : the corresponding output works.

The condition $\phi_{2}>\phi_{1}$ leads $V_{d}<0$, and it establishes a positive potential difference between the semiconductor with low work function and the semiconductor with high work function.

The differences in density of states and semiconductor doping carry different values of the parameters $e \phi_{F_{1}}$ and $e \phi_{F_{2}}$, so different values of the conduction bands energies of the two neutral regions of the semiconductors that is [12]:

$$
\Delta E_{c_{n}}=\Delta E_{c_{1}}-\Delta E_{c_{2}}=e\left(\phi_{F_{1}}-\phi_{F_{2}}\right),
$$

where $e \phi_{F_{1}}=E_{c_{1}}-E_{F_{1}}$ and $e \phi_{F_{2}}=E_{c_{2}}-E_{F_{2}}$ with $\Delta E_{c_{n}}$ : the energy difference of the conduction bands; $\Delta E_{v_{n}}$ : the energy difference of the valence bands; $\Delta E_{g}$ : the gaps 


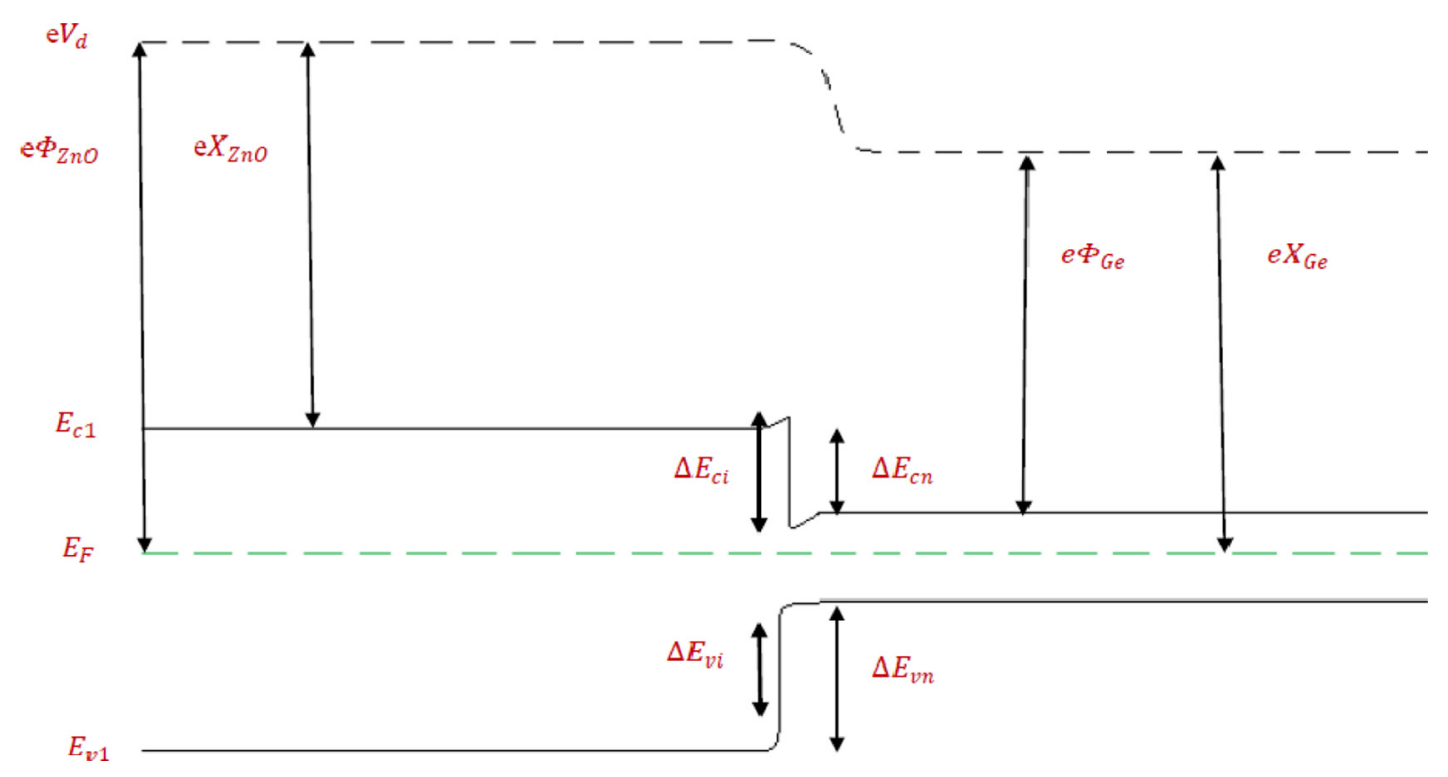

Fig. 6. Diagram of energy bands structure of $\mathrm{ZnO} / \mathrm{n}$-type germanium.

difference of the two semiconductors; $E_{c_{i}}$ : the conduction band energies of the semiconductor $i(i=1 ; 2) ; E_{F_{i}}$ : the fermi energies of the semiconductor $i(i=1 ; 2)$.

Finally, if the gap difference of the energies of the two semiconductors is different from $\Delta E_{c_{n}}$, the complement is expressed by an energy difference $E_{g}$ between the valence bands and the conduction bands:

$$
\begin{gathered}
\Delta E_{g}=E_{g_{2}}-E_{g_{1}}=\left(E_{c_{2}}-E_{c_{1}}\right)-\left(E_{v_{2}}-E_{v_{1}}\right), \\
\text { So : } \Delta E_{g}=\Delta E_{c_{n}}-\Delta E_{v_{n}}
\end{gathered}
$$

with $\Delta E_{v_{n}}$ : the energy difference of the valence bands; $\Delta E_{g}$ : the gaps difference of the two semiconductors.

The energy difference of the valence bands is therefore given by:

$$
\Delta E_{v_{n}}=e\left(\phi_{F_{2}}-\phi_{F_{1}}\right)-\Delta E_{g}
$$

Moreover

$$
\begin{aligned}
\Delta E_{c_{n}} & =e\left(\phi_{F_{2}}-\phi_{F_{1}}\right)=e\left[\left(\phi_{2}-\chi_{2}\right)-\left(\phi_{1}-\chi_{1}\right)\right] \\
& =-e V_{d}-e\left(\chi_{2}-\chi_{1}\right)
\end{aligned}
$$

$$
\text { So : } \Delta E_{v_{n}}=-e V_{d}-\left[e\left(\chi_{2}-\chi_{1}\right)+\Delta E_{g}\right] \text {, }
$$

with $e \chi_{1}$ : the electronic affinity of the semiconductor $1 ; e \chi_{2}$ : the electronic affinity of the semiconductor 2 .

Due to the continuity of the potential at the interface $V_{1}=V_{2}$ and therefore the equations 4 and 5 become in the vicinity of the junction:

$$
\begin{gathered}
\Delta E_{c_{n}}(\text { at the interface })=-e\left(\chi_{2}-\chi_{1}\right), \\
\Delta E_{v_{n}}(\text { at the interface })=-\left[e\left(\chi_{2}-\chi_{1}\right)+\Delta E_{g}\right] .
\end{gathered}
$$

\subsection{Heterojunction $n-n$ : zinc oxide-germanium}

Our hetero structure corresponds to a junction of zinc oxide on the n-type front of a poly-mirror germanium photodiode where $e \phi_{\mathrm{ZnO}}>e \phi_{\mathrm{Ge}}$.

Thus, the electrons diffuse from the germanium to the zinc oxide and the holes from the zinc oxide to the germanium. The state of equilibrium is established by diffusion so the electrons are repelled from the interface and the holes are drawn.

We have: $E_{\mathrm{ZnO}}=3.33 \mathrm{eV}$ [4], $\quad \chi_{\mathrm{ZnO}}=4.1 \mathrm{eV}$ [12], $e \phi_{\mathrm{ZnO}}=5.33 \mathrm{eV}$ [13], $E_{\mathrm{Ge}}=0.66 \mathrm{eV}$ [13], $e \chi_{\mathrm{Ge}}=4 \mathrm{eV}$ [14], $e \phi_{\mathrm{Ge}}=4.16 \mathrm{eV}$ [14]. $\mathrm{eV}$.

When $e V_{d}=e\left(V_{2}-V_{1}\right)=-\left(e \phi_{\mathrm{Ge}}-e \phi_{\mathrm{ZnO}}\right)=-1.17$

In the vicinity of the junction we have:

$$
\begin{aligned}
& -e\left(\chi_{\mathrm{Ge}}-\chi_{\mathrm{ZnO}}\right)=0.1 \mathrm{eV}, \\
& \Delta E_{g}=E_{\mathrm{Ge}}-E_{\mathrm{ZnO}}=-2.67 \mathrm{eV} \\
& -\left[\Delta E_{g}+e\left(\chi_{\mathrm{Ge}}-\chi_{\mathrm{ZnO}}\right)\right]=2.77 \mathrm{eV}
\end{aligned}
$$

Therefore

$$
\Delta E_{c_{n}}=-\left[e\left(\phi_{\mathrm{Ge}}-\phi_{\mathrm{ZnO}}\right)-e\left(\chi_{\mathrm{Ge}}-\chi_{\mathrm{ZnO}}\right)\right]=-1.27 \mathrm{eV},
$$

$$
\Delta E_{v_{n}}=-\left[\Delta E_{g}+e\left(\chi_{\mathrm{Ge}}-\chi_{\mathrm{ZnO}}\right)\right]-e V_{d}=3.94 \mathrm{eV} .
$$

From the various explanations, we can propose as an energy diagram of the first part of our designed structure so $\mathrm{ZnO}$ /front germanium photodiode, the following diagram (Fig. 6).

Following the diffusion of electrons from $\mathrm{Ge}$ to $\mathrm{ZnO}$ and holes from $\mathrm{ZnO}$ to $\mathrm{Ge}$, it appears a positive space charge zone in the $\mathrm{Ge}$ and a negative one in $\mathrm{ZnO}$. The nature and extent of the space charge zone depends on the type of each semiconductor. 


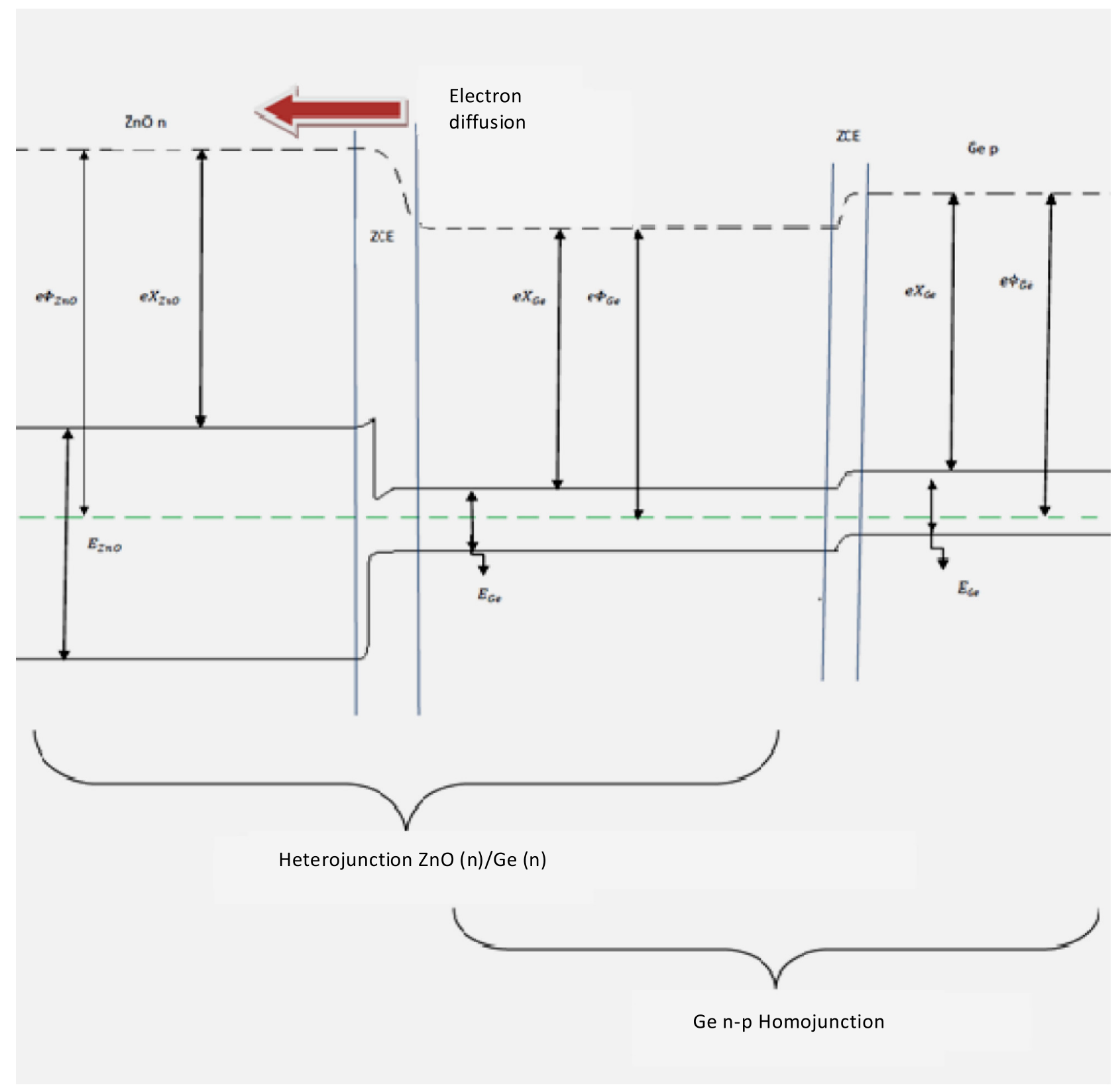

Fig. 7. Energy band diagram of the entire $\mathrm{ZnO} /$ Ge photodiode.

In the $\mathrm{ZnO}$ semiconductor the space charge zone is negative. It is due to an increase in electron density in the vicinity of the interface. Hence the space charge in the $\mathrm{ZnO}$ semiconductor is an accumulating charge and it is located in the immediate vicinity of the interface. For the Ge semiconductor the space charge zone is at contrary positive. It is established in the vicinity of the interface a depletion regime with a certain spatial extension of the charge density.

\subsection{Final diagram of the $\mathrm{ZnO} / \mathrm{Ge}$ photodiode}

The structure of our $\mathrm{ZnO} / \mathrm{Ge}$ photodiode consists of two parts. The first part is an heterojunction $n-n$ zinc oxide and germanium. The second part is a $n-p$ homojunction germanium which formed the basis of photodiode before the deposition of the $\mathrm{ZnO}$ layer.
Taking into account all the characteristic parameters of all parts of the $\mathrm{ZnO} / \mathrm{Ge}$ photodiode, we propose in Figure 7 an energy bands diagram of the structure.

The current is still more important at a strong discontinuity than at the level of a pseudo-continuity. Near to the strong discontinuity, the potential barrier is relatively low near the top so some carriers can pass through it by Tunnel effect. This process can be described by an intra-band process [12].

\section{Conclusion}

The electrical and optical studies on the $\mathrm{ZnO} / \mathrm{Ge}$ photodiode, demonstrate the significant improvement of germanium junctions after introduction of thin aluminum- 
doped $\mathrm{ZnO}$ layers in the structure. Indeed, we have noted a reduction in the reflectivity of $13 \%$. The new photodiode has a reflectivity of some $21 \%$ on average over the wavelength range of $800-2000 \mathrm{~nm}$. We also highlighted the improvement of the electrical characteristics particularly the shunt resistance which climbed from $95 \Omega$ up to $12.915 \mathrm{k} \Omega$ with the deposit of the thin $\mathrm{ZnO}$ layer. Nevertheless we have noted a slight increase in the series resistance, which rose from $1.08 \Omega$ to $36 \Omega$ but still comparable to the commercial photodiodes. The proposed energy band diagram explains the transfer process of carriers charge in the different sections of our $\mathrm{ZnO} / \mathrm{Ge}$ structure. It also brings some additional physical arguments to the observed electrical improvements. We plan to further work in order to lead a metrological study of the performed photodiode. This will eventually help to design this type of photodiode for radiometric applications in our metrology laboratory.

\section{References}

1. E. Akkari, O. Touayar, F.J. del Campo, J. Monteserrat, Improved electrical characteristics of porous germanium photodiode obtained by phosphorus ion implantation, Int. J. Nanotechnol. 10, 553-562 (2013)

2. E. Akkari, Z. Ben Achour, S. Aouida, O. Touayar, B. Bessais, J. Ben Brahim, Study and characterization of porous germanium for radiometric measurements, Phys. Status Solidi C 6, 1685-1688 (2009)

3. E. Akkari, O. Touayar, B. Bessais, Reflectivity, absorption and structural studies of porous germanium, Sensor Lett. 9, 2295-2298 (2011)
4. Z. Ben Achour, T. Ktari, B. Ouertani, O. Touayar, B. Bessais, J. Ben Brahim, Effect of doping level and spray time on zinc oxide thin films produced by spray pyrolysis for transparent electrodes applications, Sens. Actuators A 134, 447-451 (2007)

5. S. Major, S. Kumar, M. Bhatnagar, Appl. Phys. Lett. 49, 394 (1986)

6. Z. Ben Achour, O. Touayar, E. Akkari, J. Bastie, B. Bessais, J. Ben Brahim, Study and realization of a transfer detector based on porous silicon for radiometric measurements, Nucl. Instrum. Methods Phys. Res. A 579, 1117-1121 (2007)

7. F. Paraguay, W. Estrada, D.R. Acosta, Thin Solid Films 350, 192 (1999)

8. M. Amlouk, F. Touhari, S. Belgacem, N. Kamoun, R. Bennaceur, Phys. Status Solidi (a) 163, 73 (1997)

9. E. Simoen, A. Satta, A. D'Amore, T. Janssens, T. Clarysse, K. Martens, B. De Jaeger, A. Benedetti, I. Hoflijk, B. Borijs, M. Meuris, W. Vandercost, Mater. Sci. Semicond. Process. 9, 634-639 (2006)

10. Th. Canneaux, D. Mathiot, J.P. Ponpon, S. Roques, S. Schmitt, Ch. Dubois, Mater. Sci. Eng. B 154-155, 68-71 (2008)

11. Z. Ben Achour, O. Touayar, N. Sifi, Stability study of the metrological characteristics of a $\mathrm{ZnO} / \mathrm{PS} / \mathrm{C}-\mathrm{Si}$ photodiode (PSiZ) used as a transfer standard in the visible spectral range, Mod. Instrum. 1, 21-25 (2012)

12. H. Mathieu, Physique des semi-conducteurs et des composants électronique (Edition Masson, 2009), pp. 300-334

13. S.Yu. Davydov, V.A. Moshnikov, A.A. Fedotov, Gas adsorption on semiconducting oxides: a change in the work function, Tech. Phys. Lett. 30, 727-729 (2004)

14. Handbook of semiconductor germanium technology/germanium based from materials to devices, Vol. 4, 2nd edition, Methods A 518, 775 (2004)

Cite this article as: : Zahra Ben Achour, Marwa Hammami, Oualid Touayar, Development and characterization of a ZnO/Ge photodiode for optical radiation measurements in the near infrared, Int. J. Metrol. Qual. Eng. 8, 24 (2017) 